\title{
Psychiatric comorbidity and psychosocial impairment among patients with vertigo and dizziness
}

\author{
Claas Lahmann, ${ }^{1,2}$ Peter Henningsen, ${ }^{1,2}$ Thomas Brandt, ${ }^{2,3}$ Michael Strupp, ${ }^{2,4}$ \\ Klaus Jahn, ${ }^{2,4}$ Marianne Dieterich, ${ }^{2,4,5}$ Annegret Eckhardt-Henn, ${ }^{6}$ \\ Regina Feuerecker, ${ }^{2,4}$ Andreas Dinkel, ${ }^{1}$ Gabriele Schmid ${ }^{1,2}$
}

\begin{abstract}
- Additional material is published online only. To view please visit the journal online (http://dx.doi.org/10.1136/ jnnp-2014-307601).

${ }^{1}$ Department of Psychosomatic Medicine and Psychotherapy, Klinikum rechts der Isar, Technische Universität München, Munich, Germany ${ }^{2}$ German Center for Vertigo and Balance Disorders, University Hospital Munich, Campus Großhadern, LudwigMaximilians-Universität, Munich, Germany ${ }^{3}$ Department of Clinical Neuroscience, University Hospital Munich, Campus Großhadern, LudwigMaximilians-Universität, Munich, Germany

${ }^{4}$ Department of Neurology, University Hospital Munich, Campus Großhadern, LudwigMaximilians-Universität, Munich, Germany ${ }^{5}$ Munich Cluster of Systems Neurology (SyNergy), Munich, Germany

${ }^{6}$ Department of Psychosomatic Medicine and Psychotherapy, Klinikum Stuttgart, Stuttgart, Germany
\end{abstract}

\section{Correspondence to} Dr Claas Lahmann, Department of Psychosomatic Medicine and Psychotherapy, Klinikum rechts der Isar, Technische Universität München, Langerstr. 3, Münich 81675, Germany; lahmann@tum.de

Received 8 January 2014 Revised 29 May 2014 Accepted 6 June 2014 Published Online First 24 June 2014

\section{CrossMark}

To cite: Lahmann $C$ Henningsen $P$, Brandt T, et al. I Neurol Neurosurg Psychiatry 2015;86: 302-308.

\section{ABSTRACT}

Background Vertigo and dizziness are often not fully explained by an organic illness, but instead are related to psychiatric disorders. This study aimed to evaluate psychiatric comorbidity and assess psychosocial impairment in a large sample of patients with a wide range of unselected organic and non-organic (ie, medically unexplained) vertigo/dizziness syndromes. Methods This cross-sectional study involved a sample of 547 patients recruited from a specialised interdisciplinary treatment centre for vertigo/dizziness. Diagnostic evaluation included standardised neurological examinations, structured clinical interview for major mental disorders (SCID-I) and self-report questionnaires regarding dizziness, depression, anxiety, somatisation and quality of life.

Results Neurological diagnostic workup revealed organic and non-organic vertigo/dizziness in $80.8 \%$ and $19.2 \%$ of patients, respectively. In $48.8 \%$ of patients, SCID-I led to the diagnosis of a current psychiatric disorder, most frequently anxiety/phobic, somatoform and affective disorders. In the organic vertigo/dizziness group, $42.5 \%$ of patients, particularly those with vestibular paroxysmia or vestibular migraine, had a current psychiatric comorbidity. Patients with psychiatric comorbidity reported more vertigo-related handicaps, more depressive, anxiety and somatisation symptoms, and lower psychological quality of life compared with patients without psychiatric comorbidity.

Conclusions Almost half of patients with vertigo/ dizziness suffer from a psychiatric comorbidity. These patients show more severe psychosocial impairment compared with patients without psychiatric disorders. The worst combination, in terms of vertigo-related handicaps, is having non-organic vertigo/dizziness and psychiatric comorbidity. This phenomenon should be considered when diagnosing and treating vertigo/ dizziness in the early stages of the disease.

\section{INTRODUCTION}

Vertigo and dizziness, which have a lifetime prevalence of approximately $30 \%,{ }^{1}$ are common symptoms presented to general practitioners and neurologists. $^{2} 3$ In approximately $30-50 \%$ of patients with vertigo/dizziness, complaints are not fully explained by a vestibular deficit or a defined organic illness, but instead are related to psychiatric disorders. ${ }^{45}$ In a sample of 189 patients with complaints of vertigo/dizziness, $52.4 \%$ had medically unexplained symptoms. In this group, vertigo/ dizziness was often associated with anxiety/phobic (45.5\%), somatoform (41.4\%) or depressive disorders $(13.1 \%){ }^{4}$

Psychiatric comorbidity seems to be more prevalent in certain subgroups of organic vertigo/dizziness. ${ }^{6}$ For example, Eckhardt-Henn $e t a l^{7}$ found that in a sample of 68 patients with vertigo/dizziness, those who suffered from vestibular migraine or Meniere's disease had significantly higher rates of psychiatric comorbidity $(65 \%$ and $57 \%$, respectively), particularly anxiety and depressive disorders, compared with patients who suffered from vestibular neuritis $(22 \%)$ or benign paroxysmal positional vertigo (BPPV) $(15 \%)$. In a study of 59 patients with vestibular disorders, patients with vestibular migraine had the highest chance of psychiatric comorbidity at a 1 -year follow-up $(58.5 \%){ }^{8}$ Thus, prognosis may be poor in patients who suffer from severe and relapsing vertigo attacks. Additional risk factors may be negative beliefs about the consequences of vertigo/dizziness, anxiety/panic-related cognition or maladaptive coping strategies (eg, catastrophic thoughts, tendency to evaluate body sensations fearfully). By contrast, psychological well-being and resilient coping protect against the development of psychiatric comorbidity. ${ }^{5-10}$

Approximately $80 \%$ of patients with vertigo/dizziness with a comorbid psychiatric disorder report lower quality of life (QoL) than those without any psychiatric comorbidity. These patients feel more impaired in their daily lives or are unable to work due to their vertigo/dizziness symptoms. Moreover, patients with vertigo/dizziness with a comorbid psychiatric disorder frequently use the healthcare system. $^{8}{ }^{11}$ Given patients' vertigo/dizziness-related handicaps and associated costs for healthcare and social systems, it is important to identify and effectively treat these patients as early as possible.

Although previous studies show associations between selected subgroups of patients with vertigo/ dizziness and psychiatric comorbidity, ${ }^{4} 69$ these studies are based on small sample sizes and focus only on selected organic vertigo/dizziness groups, such as patients with vestibular migraine, Meniere's disease, BPPV or vestibular neuritis. Here we evaluated psychiatric comorbidity in a large, nonpreselected sample of patients with either nonorganic or organic forms of vertigo/dizziness, including diverse and incompletely explored organic disorders (eg, vestibular paroxysmia). Moreover, we investigated psychosocial impairment 
within these patients via self-report questionnaires on vertigo-related handicaps and symptoms, anxiety, depression, somatisation, and health-related QoL (HRQoL). Our hypotheses were that (1) particular psychiatric comorbidities are more prevalent for specific vestibular disorders, and (2) patients with psychiatric comorbidity have greater psychosocial handicaps and impairment compared with those without mental disorders after controlling for age, sex and duration of vertigo/dizziness.

\section{PATIENTS AND METHODS}

\section{Study design and sample}

This cross-sectional study, conducted between May 2010 and June 2012, involved 687 patients who gave informed consent (from a total of 860 eligible patients). Reasons for refusal were insufficient language skills, a lack of interest, blindness, cognitive difficulties in filling out a questionnaire, feelings of excessive demands or privacy concerns. Patients were recruited through routine care appointments at the German Centre for Vertigo and Balance Disorders at the University Hospital Munich, Campus Großhadern. For organisational reasons (eg, living outside of Munich or vomiting after caloric testing), not all patients underwent a Structured Clinical Interview (SCID-I) to assess mental disorders. ${ }^{12}$ Therefore, we only included and analysed data from the 547 patients $(44.1 \%$ male, $54.8 \pm 16.0$ years of age) who were interviewed. There were no significant differences between patients who did and did not participate in the SCID-I concerning age, sex, vertigo severity, vertigo-related handicap, depression, anxiety, somatisation, or physical or psychological HrQoL.

Nearly all $(91.5 \%)$ patients suffered from vertigo/dizziness for at least 3 months; $46.2 \%$ had vertigo/dizziness symptoms for more than 3 months up to 2 years, and $45.3 \%$ had symptoms for more than 2 years. Furthermore, $26.1 \%$ of patients had taken medication $(43.2 \%$ of patients with Meniere's disease received Betahistin), $7.5 \%$ practised liberatory manoeuvres, $4.8 \%$ received physiotherapeutic treatment, $4.2 \%$ had psychotherapy and $0.7 \%$ underwent surgery.

Patients were included if they were at least 18 years of age and possessed sufficient German language skills. Exclusion criteria were any of the following diagnoses: neurodegenerative disorder (eg, dementia), schizoaffective or psychotic disorder, substance abuse or severe suicidal tendencies. All patients were informed about the aims of the study and gave written informed consent. This study was approved by the Ethics Committee of the University of Munich (ref. 108-10). ${ }^{13}$

\section{Assessment and psychological instruments \\ Neurological diagnostic workup}

All patients underwent structured history-taking and a systematic and standardised physical examination by an expert medical scientist at the German Centre for Vertigo and Balance Disorders, including complete neurological, neuro-otological and neuro-ophthalmological examinations. Vestibular testing included the head impulse test, measurement of subjective visual vertical and ocular torsion, and caloric irrigation by video-oculography. The expert medical scientist made a clinical diagnosis based on the results of examinations according to diagnostic criteria. ${ }^{14}$ The diagnosis of vestibular migraine was based on criteria from Neuhauser and Lempert, ${ }^{15} 16$ the diagnosis of Meniere's disease was based on criteria from the American Academy of Otolaryngology, Head, and Neck Surgery, ${ }^{17}$ and the diagnosis of vestibular paroxysmia, which is attributed to neurovascular cross-compression and leads to vertiginous spells often accompanied by unsteadiness in stance or gait, was based on criteria from Brandt and Dieterich ${ }^{18}$ and Hüfner et al. ${ }^{19}$ The co-occurrence of multiple organic vertigo/dizziness diagnoses was possible. Based on the neurological diagnostic workup, the following two groups emerged:

1. Patients with an organic or vestibular cause of vertigo/dizziness (ie, the presence of one or more of the above mentioned diagnoses, including those patients with a combination of organic and non-organic causes of dizziness).

2. Patients with a non-organic or non-vestibular cause of vertigo/ dizziness (ie, medically unexplained vertigo/dizziness).

\section{Psychometric examination: Structured Clinical Interview (SCID-I)}

Clinical staff conducted SCID-I interviews to assess patients' psychiatric disorders according to the Diagnostic and Statistical Manual of Mental Disorders, Fourth Edition (DSM-IV) classification system. ${ }^{12}$ The co-occurrence of multiple psychiatric diagnoses was possible. All interviewers were initially blind to the organic vs non-organic vertigo/dizziness diagnoses of patients, that is, the SCID-I interviewers were not informed about any potential diagnoses of these patients. If the interviewer suspected a somatoform disorder due to vertigo/dizziness, this diagnosis was assigned only if the patient had been classified as having non-organic vertigo/dizziness. All interviewers underwent intensive training, including practice interviews with patients who were not recruited for the study. Inter-rater reliability was evaluated via interviews with a simulated patient; the $\kappa$ value was 0.94 . Interviewers were required to undergo SCID-I supervision led by a senior physician on a regular basis (ie, every 3-4 weeks).

\section{Patient self-report questionnaires}

The Vertigo Handicap Questionnaire, ${ }^{20}$ the Vertigo Symptom Scale, ${ }^{21}$ the revised Beck Depression Inventory, ${ }^{22}$ the Beck Anxiety Inventory, ${ }^{23}$ the Patient Health Questionnaire ${ }^{24}$ and the Short-Form Health Survey (SF-12) on HRQoL ${ }^{25}$ were completed by patients (table 1 ).

\section{Statistical analysis}

Statistical analysis was conducted using SPSS (V.21.0). To test for differences among SCID-I participants and non-participants, independent samples t tests were conducted; because of inflation of $\alpha$ due to multiple comparisons, statistical significance level was set at $p<0.006(0.05 / 8)$. To test for differences in selfreport questionnaire scores among vertigo/dizziness subgroups, we computed descriptive statistics and performed $\chi^{2}$ tests and analyses of variance controlled for sex, age and duration of vertigo/dizziness. Due to inflation of $\alpha$, statistical significance level was set at $\mathrm{p}<0.002(0.05 / 28)$ for $\chi^{2}$ tests and $\mathrm{p}<0.01$ $(0.05 / 8)$ for analyses of variance. When statistically significant main effects were detected, we conducted Bonferroni post hoc tests controlled for age and sex. Effect sizes (partial $\eta^{2}$ ) were computed and considered small if partial $\eta^{2}$ was $\geq 0.01$ and $<0.06$, medium if partial $\eta^{2}$ was $\geq 0.06$ and $<0.14$, and large if partial $\eta^{2}$ was $\geq 0.14 .^{26}$ To examine the relationship between self-report questionnaire scores and the occurrence of psychiatric comorbidity, we conducted binary logistic regression analysis controlled for sex, age and duration of vertigo/dizziness. The fit of the model was evaluated using the omnibus test of coefficients $\left(\chi^{2}\right.$, statistically significant if $\left.\mathrm{p}<0.05\right)$ and the Hosmer-Lemeshow statistic $\left(\chi^{2}\right.$, good fit if $\left.p>0.05\right) .{ }^{27}$

\section{RESULTS}

\section{Neurological diagnosis}

Neurological diagnostic workup revealed organic vertigo/dizziness in $442(80.8 \%)$ patients and non-organic (ie, medically 


\section{Neuropsychiatry}

Table 1 Overview and description of patients' self-report questionnaire scores

\begin{tabular}{|c|c|c|c|c|}
\hline Questionnaire & Description & Scores, scales & 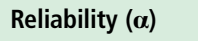 & Validity \\
\hline $\begin{array}{l}\text { Vertigo Handicap Questionnaire } \\
\text { (VHQ) }\end{array}$ & $\begin{array}{l}\text { Assessment of physical and psychosocial } \\
\text { handicap due to vertigo/dizziness } \\
\text { - } 25 \text { items } \\
\text { - Five-point scale: } 0 \text { (never) to } 4 \text { (always) }\end{array}$ & Sum score ranging from 0 to 100 & High $(\alpha=0.92)$ & $\begin{array}{l}\text { Good } \\
\text { construct } \\
\text { validity }\end{array}$ \\
\hline Vertigo Symptom Scale (VSS) & $\begin{array}{l}\text { Assessment of frequency of dizziness-related } \\
\text { symptoms } \\
\text { } 34 \text { items } \\
\text { Five-point scale: } 0 \text { (never) to } 4 \text { (more than once } \\
\text { per week) }\end{array}$ & $\begin{array}{l}\text { Two subscales: (I) vertigo and associated } \\
\text { symptoms (VSS-VER) and (II) somatic } \\
\text { anxiety and autonomic arousal (VSS-AA) }\end{array}$ & $\begin{array}{l}\text { Good (VSS-VER } \\
\alpha=0.79 ; \text { VSS-AA } \\
\alpha=0.89 \text { ) }\end{array}$ & $\begin{array}{l}\text { Good } \\
\text { construct } \\
\text { validity }\end{array}$ \\
\hline $\begin{array}{l}\text { Beck Depression Inventory, } \\
\text { revised (BDI-2) }\end{array}$ & $\begin{array}{l}\text { Assessment of depression severity during the last } \\
2 \text { weeks } \\
\text { - } 21 \text { items } \\
\text { Four-point scale: } 0-3\end{array}$ & $\begin{array}{l}\text { Sum score ranging from } 0 \text { to } 63: \leq 8 \text {, not } \\
\text { clinically relevant; } 9-13 \text {, subclinical } \\
\text { depression; } 14-19 \text {, } \\
\text { mild depression; } 20-28, \\
\text { moderate depression; } \geq 29 \text {, } \\
\text { severe depression }\end{array}$ & Good $(\alpha \geq 0.80)$ & $\begin{array}{l}\text { Good } \\
\text { construct } \\
\text { validity }\end{array}$ \\
\hline Beck Anxiety Inventory (BAI) & $\begin{array}{l}\text { Assessment of anxiety severity during the last } \\
7 \text { days } \\
\text { - } 21 \text { items } \\
\text { Four-point scale: } 0 \text { (not at all) to } 3 \text { (strongly) }\end{array}$ & $\begin{array}{l}\text { Sum score ranging from } 0 \text { to } 63: \leq 7 \text {, not } \\
\text { clinically relevant; } 8-15 \text {, mild anxiety; } \\
16-25 \text {, moderate anxiety; } \geq 26 \text {, clinically } \\
\text { relevant anxiety }\end{array}$ & Good $(\alpha \geq 0.085)$ & $\begin{array}{l}\text { Good } \\
\text { construct } \\
\text { validity }\end{array}$ \\
\hline $\begin{array}{l}\text { Patient Health Questionnaire } \\
\text { (PHQ-15) }\end{array}$ & $\begin{array}{l}\text { Assessment of most important Diagnostic and } \\
\text { Statistical Manual of Mental Disorders, Fourth } \\
\text { Edition (DSM-IV) criteria for somatisation } \\
\text { disorders, and two items from the depression } \\
\text { module } \\
15 \text { items } \\
\text { Three-point scale: } 0 \text { (not bothered at all) to } 2 \\
\text { (bothered a lot/more than half of the time or } \\
\text { nearly every day) }\end{array}$ & $\begin{array}{l}\text { Sum score ranging from } 0 \text { to } 30: \leq 4 \text {, } \\
\text { minimal; } 5-9 \text {, low; } 10-14 \text {, medium; } \geq 15 \text {, } \\
\text { high }\end{array}$ & Good $(\alpha=0.080)$ & $\begin{array}{l}\text { Good } \\
\text { construct } \\
\text { validity }\end{array}$ \\
\hline $\begin{array}{l}\text { Health-related quality of life } \\
\text { measure (HRQoL) Short-Form } \\
\text { Health Survey (SF-12) }\end{array}$ & $\begin{array}{l}\text { Assessment of HRQoL during the last } 4 \text { weeks } \\
12 \text { items }\end{array}$ & $\begin{array}{l}\text { Physical and psychological HRQoL, } \\
\text { ranging from } 0 \text { (very poor) to } 100 \\
\text { (optimal) }\end{array}$ & $\begin{array}{l}\text { Moderate to good } \\
(\alpha \geq 0.070)\end{array}$ & $\begin{array}{l}\text { Good } \\
\text { convergent } \\
\text { validity }\end{array}$ \\
\hline
\end{tabular}

unexplained) vertigo/dizziness in 105 (19.2\%) patients. Among patients with organic vertigo/dizziness, vestibular migraine was the most frequent diagnosis $(n=95)$, followed by BPPV $(n=87)$, Meniere's disease $(n=81)$, bilateral vestibulopathy $(n=45)$, vestibular paroxysmia $(n=43)$, multisensory deficit $(n=42)$, central vertigo $(n=38)$, vestibular neuritis $(n=29)$, polyneuropathy $(n=26)$ and unilateral vestibular loss $(n=20)$. Although most (75.1\%) patients had a single organic vertigo/dizziness diagnosis, $20.6 \%$ had two diagnoses, 3.6\% had three diagnoses and $0.7 \%$ had four diagnoses.

\section{Prevalence of psychiatric comorbidity}

Next, we analysed the prevalence of current psychiatric comorbidity. SCID-1 using DSM-IV criteria led to a diagnosis of current mental disorder in $48.8 \%$ of patients. Specifically, $158(28.9 \%)$ patients had an anxiety/phobic disorder, 136 (24.9\%) had a somatoform disorder, 104 (19.0\%) had an affective disorder, $16(2.9 \%)$ had a substance abuse disorder and 4 $(0.7 \%)$ had an eating disorder. Some patients had more than one psychiatric comorbidity; 42 patients (7.7\%) had anxiety/ phobic and somatoform disorders, 32 (5.9\%) had anxiety/ phobic and affective disorders, 21 (3.8\%) had affective and somatoform disorders and 20 (3.7\%) had anxiety/phobic, affective and somatoform disorders.

Among patients with current anxiety/phobia, the most frequent diagnosis was specific phobia $(n=61)$, primarily of heights, flying, or small and tight rooms, followed by agoraphobia without panic disorder $(\mathrm{n}=53)$, panic disorder with agoraphobia $(n=33)$, panic disorder without agoraphobia $(n=24)$, generalised anxiety disorder $(n=16)$ and social phobia $(n=10)$.

\section{Combination of neurological and psychiatric diagnoses}

When considering neurological diagnoses and psychiatric comorbidities, four subgroups emerged: patients with organic vertigo/dizziness and no psychiatric comorbidity (group $\mathrm{o}^{+} / \mathrm{p}^{-}$), patients with non-organic vertigo/dizziness and no psychiatric comorbidity (group $\mathrm{o}^{-} / \mathrm{p}^{-}$; ie, patients with disturbed well-being on a subclinical level), patients with organic vertigo/dizziness and psychiatric comorbidity (group $\mathrm{o}^{+} / \mathrm{p}^{+}$), and patients with non-organic vertigo/dizziness and psychiatric comorbidity (group $\mathrm{o}^{-} / \mathrm{p}^{+}$). The prevalence of psychiatric disorders was significantly higher $\left(\chi^{2}=45.9 ; \mathrm{df}=1 ; \mathrm{p}<0.001\right)$ among patients with non-organic vertigo/dizziness $(75.2 \%)$ than among patients with organic vertigo/dizziness $(42.5 \%)$ (table 2$)$.

Most patients in subgroups $\mathrm{o}^{+} / \mathrm{p}^{-}(48.7 \%), \mathrm{o}^{-} / \mathrm{p}^{-}(60.9 \%)$, and $\mathrm{o}^{+} / \mathrm{p}^{+}(44.7 \%)$ suffered from vertigo/dizziness for between 3 months and 2 years, whereas most patients in subgroup $\mathrm{o}^{-} / \mathrm{p}^{+}$ had vertigo/dizziness for 2-10 years (46.3\%). Furthermore, age and sex differed significantly among the four subgroups (see online supplementary table A1).

\section{Psychiatric comorbidity in organic vertigo/dizziness subgroups}

In the organic vertigo/dizziness subgroup, 22 (51.2\%) patients with vestibular paroxysmia and $47(49.5 \%)$ patients with vestibular migraine had a current psychiatric comorbidity, whereas only $11(24.4 \%)$ of patients with bilateral vestibulopathy fulfilled criteria for a current comorbid mental disorder. Anxiety/phobic disorders were most frequent in patients with vestibular paroxysmia $(32.6 \%)$ or vestibular migraine $(32.6 \%)$ and least frequent in patients with bilateral vestibulopathy 
Table 2 Combination of neurological diagnosis with psychiatric comorbidity $(n=547)$

\begin{tabular}{|c|c|c|c|}
\hline & \multicolumn{2}{|l|}{ SCID-I } & \\
\hline & No psychiatric comorbidity & Psychiatric comorbidity & \\
\hline \multicolumn{4}{|l|}{ Neurological diagnosis } \\
\hline Organic vertigo/dizziness & $254(57.5 \%)\left(\right.$ group o $\left./ \mathrm{p}^{-}\right)$ & $188(42.5 \%)\left(\right.$ group o ${ }^{+} / \mathrm{p}^{+}$) & $442(80.8 \%)$ \\
\hline Non-organic (ie, medically unexplained) vertigo/dizziness & $26(24.8 \%)\left(\right.$ group o $\left./ \mathrm{p}^{-}\right)$ & $79(75.2 \%)\left(\right.$ group o- $\left./ \mathrm{p}^{+}\right)$ & $105(19.2 \%)$ \\
\hline \multicolumn{4}{|c|}{$\begin{array}{l}\text { Group } 0^{+} / \mathrm{p}^{-} \text {, organic vertigo/dizziness without psychiatric comorbidity. } \\
\text { Group } 0^{-} / \mathrm{p}^{-} \text {, non-organic vertigo/dizziness without psychiatric comorbidity. } \\
\text { Group } 0^{+} / \mathrm{p}^{+} \text {, organic vertigo/dizziness with psychiatric comorbidity. } \\
\text { Group } 0^{-} / \mathrm{p}^{+} \text {, non-organic vertigo/dizziness with psychiatric comorbidity. }\end{array}$} \\
\hline
\end{tabular}

$(17.8 \%)$ or vestibular neuritis (17.2\%). Affective disorders occurred most frequently in patients with central vertigo $(23.7 \%)$ or BPPV $(23.0 \%)$ and occurred least frequently in patients with bilateral vestibulopathy $(11.1 \%)$. Somatoform disorders were most frequent in patients with vestibular migraine $(21.1 \%)$ and least frequent in patients with bilateral vestibulopathy $(8.9 \%)$ (table 3$)$.

\section{Differences in psychosocial impairment assessed by self-report questionnaires}

Overall, there were significant differences in subjective psychosocial impairment and handicaps between patients with and without psychiatric comorbidity (table 4). That is, compared with patients with psychiatric comorbidity, patients with no psychiatric comorbidity reported less vertigo-related handicaps, fewer vertigo-related symptoms, less autonomic arousal, fewer depressive, anxiety and somatisation symptoms, and higher psychological HRQoL after controlling for age, sex and duration of vertigo/dizziness. Effect sizes were clinically relevant (table 4, see online supplementary tables A2 and A3). The worst combination, in terms of vertigo-related handicaps, was having nonorganic vertigo/dizziness and psychiatric comorbidity.

Finally, we found that vertigo-associated symptoms (Vertigo Symptom Scale-VER: OR 1.8, 95\% CI (1.1 to 2.9)), depression (Beck Depression Inventory: OR 1.1, 95\% CI (1.04 to 1.2)) and anxiety (Beck Anxiety Inventory: OR 1.1, 95\% CI (1.01 to 1.1)) increased the odds of having psychiatric comorbidity after controlling for age, sex and duration of vertigo/dizziness. The fit of the model was good (omnibus test of coefficients: $\chi^{2}=79.9$; $\mathrm{df}=14$; $\mathrm{p}<0.001$; Hosmer-Lemeshow statistic: $\chi^{2}=12.3$; $\mathrm{df}=8 ; \mathrm{p}=0.14$ ).

\section{DISCUSSION}

The aim of this study was to investigate psychiatric comorbidity in a large, unselected sample of patients, who were referred to a specialised, interdisciplinary treatment centre for vertigo/dizziness, which allowed the inclusion and analysis of several previously unexplored subgroups. We hypothesised that specific vestibular disorders are associated with particular psychiatric comorbidities and that patients with mental disorders have more psychosocial handicaps and impairment compared with those without mental disorders after controlling for age, sex and duration of vertigo/dizziness.

Our results support our first hypothesis. We found that specific psychiatric comorbidity was more prevalent in patients with certain vestibular disorders. In particular, patients who suffered from episodic vertigo/dizziness, namely vestibular paroxysmia or vestibular migraine, had the highest prevalence of psychiatric disorders. The most frequent mental disorders in patients with episodic vertigo/dizziness, such as vestibular migraine, vestibular paroxysmia and Menière's disease, were phobias or anxiety, which is partly in line with previous studies. ${ }^{6} 7$ Moreover, our results point towards patients with vestibular paroxysmia as a particularly vulnerable subgroup. To our knowledge, there have been no studies to date investigating psychiatric comorbidity in patients with vestibular paroxysmia. In episodic vertigo/dizziness conditions, such as vestibular migraine and vestibular paroxysmia, patients may unexpectedly experience intense and recurrent vertigo attacks, which might be a particular burden for these patients. One hypothesis may be that patients perceive the onset and intensity of vertigo/dizziness attacks as uncontrollable, which could trigger anxiety and panic-related cognition and sometimes lead to the development of avoidance behaviour. ${ }^{9} 28$ Previous studies also suggest a link

Table 3 Current psychiatric comorbidity in patients diagnosed with organic vertigo/dizziness $(n=442)$ and differences among subgroups

\begin{tabular}{|c|c|c|c|c|c|c|c|c|c|c|}
\hline \multirow[b]{2}{*}{ SCID-I diagnosis* } & \multicolumn{8}{|c|}{ Diagnoses of organic vertigo/dizziness } & \multirow[b]{2}{*}{$\begin{array}{l}\text { Subgroup } \\
\text { differences }\end{array}$} & \multirow[b]{2}{*}{$\chi^{2} ; \mathrm{df}(p)$} \\
\hline & $\begin{array}{l}\text { VP }(n=43) \\
\text { Frequency (\%) }\end{array}$ & $\begin{array}{l}\text { VM } \\
(n=95)\end{array}$ & $\begin{array}{l}\text { MultD } \\
(n=42)\end{array}$ & $\begin{array}{l}\text { BPPV } \\
(n=87)\end{array}$ & $\begin{array}{l}\text { CV } \\
(n=38)\end{array}$ & $\begin{array}{l}\text { MD } \\
(n=81)\end{array}$ & $\begin{array}{l}\text { VN } \\
(n=29)\end{array}$ & $\begin{array}{l}\text { BV } \\
(n=45)\end{array}$ & & \\
\hline $\begin{array}{l}\text { Current psychiatric } \\
\text { comorbidity }\end{array}$ & 51.2 & 49.5 & 45.2 & 44.8 & 42.1 & 37.9 & 37.0 & 24.4 & $\begin{array}{l}\mathrm{VP}>\mathrm{BV} \\
\mathrm{VM}>\mathrm{BV} \\
\mathrm{BPPV}>\mathrm{BV}\end{array}$ & $\begin{array}{l}16.7 ; 1 \quad(<0.001) \\
32.4 ; 1(<0.001) \\
19.7 ; 1 \quad(<0.001)\end{array}$ \\
\hline Anxiety/phobic & 32.6 & 32.6 & 23.8 & 24.1 & 18.4 & 25.9 & 17.2 & 17.8 & $\begin{array}{l}V M>C V \\
V M>V N \\
V M>B V\end{array}$ & $\begin{array}{l}12.8 ; 1(<0.001) \\
15.9 ; 1(<0.001) \\
14.3 ; 1(<0.001)\end{array}$ \\
\hline Affective & 16.3 & 14.7 & 21.4 & 23.0 & 23.7 & 16.0 & 17.2 & 11.1 & $\mathrm{BPPV}>\mathrm{BV}$ & $12.5 ; 1(<0.001)$ \\
\hline Somatoform & 16.3 & 21.1 & 9.5 & 16.1 & 15.8 & 12.3 & 17.2 & 8.9 & $\begin{array}{l}\text { VM }>\text { MultD } \\
\text { VM }>\text { BV }\end{array}$ & $\begin{array}{l}14.8 ; 1(<0.001) \\
17.3 ; 1(<0.001)\end{array}$ \\
\hline
\end{tabular}

${ }^{*}$ Multiple psychiatric diagnoses were possible.

BPPV, benign paroxysmal positional vertigo; BV, bilateral vestibulopathy; CV, central vertigo; MD, Meniere's disease; MultD, multisensory deficit; VM, vestibular migraine; VN, vestibular neuritis; VP, vestibular paroxysmia. 


\section{Neuropsychiatry}

Table 4 Differences among vertigo/dizziness subgroups in self-report questionnaire results after controlling for sex, age and duration of vertigo/dizziness

\begin{tabular}{|c|c|c|c|c|}
\hline Outcome & Subgroups* & Mean $( \pm$ SD) & $F ; d f(p) \dagger$ & Post hoc comparison of subgroups $(p)$; partial $\eta^{2} \ddagger$ \\
\hline VHQ-sum score & $\begin{array}{l}0^{+} / p^{-} \\
0^{-} / p^{-} \\
0^{+} / p^{+} \\
0^{-} / p^{+}\end{array}$ & $\begin{array}{l}39.8 \pm 17.0 \\
31.9 \pm 15.8 \\
47.9 \pm 17.3 \\
50.9 \pm 15.1\end{array}$ & $12.0 ; 3(<0.001)$ & $\begin{array}{l}0^{+} / p^{-}<0^{+} / p^{+}(<0.001) ; 0.04 \\
0^{+} / p^{-}<0^{-} / p^{+}(<0.001) ; 0.07 \\
0^{-} / p^{-}<0^{+} / p^{+}(<0.001) ; 0.09 \\
0^{-} / p^{-}<0^{-} / p^{+}(<0.001) ; 0.28\end{array}$ \\
\hline VSS—vertigo and associated symptoms & $\begin{array}{l}0^{+} / p^{-} \\
0^{-} / p^{-} \\
0^{+} / p^{+} \\
0^{-} / p^{+}\end{array}$ & $\begin{array}{l}1.0 \pm 0.7 \\
0.8 \pm 0.4 \\
1.5 \pm 0.9 \\
1.5 \pm 1.0\end{array}$ & $7.1 ; 3(<0.001)$ & $\begin{array}{l}\mathrm{o}^{+} / \mathrm{p}^{-}<0^{+} / \mathrm{p}^{+}(<0.001) ; 0.07 \\
\mathrm{o}^{+} / \mathrm{p}^{-}<0^{-} / \mathrm{p}^{+}(0.008) ; 0.05 \\
0^{-} / \mathrm{p}^{-}<0^{+} / \mathrm{p}^{+}(0.003) ; 0.10 \\
0^{-} / \mathrm{p}^{-}<0^{-} / \mathrm{p}^{+}(0.004) ; 0.17\end{array}$ \\
\hline VSS-autonomic arousal & $\begin{array}{l}0^{+} / p^{-} \\
0^{-} / p^{-} \\
0^{+} / p^{+} \\
0^{-} / p^{+}\end{array}$ & $\begin{array}{l}0.9 \pm 0.6 \\
1.1 \pm 0.7 \\
1.4 \pm 0.8 \\
1.4 \pm 0.6\end{array}$ & $12.6 ; 3(<0.001)$ & $\begin{array}{l}0^{+} / \mathrm{p}^{-}<0^{+} / \mathrm{p}^{+}(<0.001) ; \underline{0.08} \\
0^{+} / \mathrm{p}^{-}<0^{-} / \mathrm{p}^{+}(<0.001) ; \underline{0.08}\end{array}$ \\
\hline BDI-2 & $\begin{array}{l}0^{+} / p^{-} \\
0^{-} / p^{-} \\
0^{+} / p^{+} \\
0^{-} / p^{+}\end{array}$ & $\begin{array}{c}8.0 \pm 5.5 \\
9.7 \pm 8.5 \\
15.3 \pm 10.0 \\
15.2 \pm 7.3\end{array}$ & $25.7 ; 3(<0.001)$ & $\begin{array}{l}0^{+} / p^{-}<0^{+} / p^{+}(<0.001) ; 0.17 \\
0^{+} / p^{-}<0^{-} / p^{+}(<0.001) ; 0.20 \\
0^{-} / p^{-}<0^{-} / p^{+}(0.004) ; 0.12\end{array}$ \\
\hline BAl & $\begin{array}{l}0^{+} / p^{-} \\
0^{-} / p^{-} \\
0^{+} / p^{+} \\
0^{-} / p^{+}\end{array}$ & $\begin{array}{c}9.6 \pm 7.2 \\
9.3 \pm 5.4 \\
17.0 \pm 10.9 \\
18.6 \pm 8.9\end{array}$ & $23.9 ; 3(<0.001)$ & $\begin{array}{l}0^{+} / p^{-}<0^{+} / p^{+}(<0.001) ; 0.13 \\
0^{+} / p^{-}<0^{-} / p^{+}(<0.001) ; 0.19 \\
0^{-} / p^{-}<0^{+} / p^{+}(0.004) ; 0.06 \\
0^{-} / p^{-}<0^{-} / p^{+}(<0.001) ; 0.20\end{array}$ \\
\hline PHQ-15 & $\begin{array}{l}0^{+} / p^{-} \\
0^{-} / p^{-} \\
0^{+} / p^{+} \\
0^{-} / p^{+}\end{array}$ & $\begin{array}{r}8.3 \pm 4.5 \\
8.1 \pm 3.6 \\
11.4 \pm 5.1 \\
11.9 \pm 4.7\end{array}$ & $14.0 ; 3(<0.001)$ & $\begin{array}{l}0^{+} / p^{-}<0^{+} / p^{+}(<0.001) ; 0.09 \\
0^{+} / p^{-}<0^{-} / p^{+}(<0.001) ; 0.10\end{array}$ \\
\hline SF-12—Physical HRQoL & $\begin{array}{l}0^{+} / p^{-} \\
0^{-} / p^{-} \\
0^{+} / p^{+} \\
0^{-} / p^{+}\end{array}$ & $\begin{array}{l}45.3 \pm 10.7 \\
45.8 \pm 10.2 \\
44.8 \pm 11.2 \\
44.1 \pm 11.3\end{array}$ & $0.6 ; 3(0.62)$ & - \\
\hline SF-12-Psychological HRQoL & $\begin{array}{l}0^{+} / p^{-} \\
0^{-} / p^{-} \\
0^{+} / p^{+} \\
0^{-} / p^{+}\end{array}$ & $\begin{array}{l}53.0 \pm 9.0 \\
52.8 \pm 11.1 \\
48.6 \pm 13.0 \\
45.8 \pm 13.0\end{array}$ & $8.6 ; 3(<0.001)$ & $\begin{array}{l}0^{+} / p^{-}>0^{+} / p^{+}(<0.001) ; 0.03 \\
0^{+} / p^{-}>0^{-} / p^{+}(<0.001) ; 0.07 \\
0^{-} / p^{-}>0^{-} / p^{+}(0.005) ; 0.08\end{array}$ \\
\hline
\end{tabular}

${ }^{*}$ Group $0^{+} / p^{-}: n=(123 ; 254)$, group $0^{-} / p^{-}: n=(14 ; 26) ;$ group $o^{+} / p^{+}: n=(79 ; 188) ;$ group $o^{-} / p^{+}: n=(38 ; 79)$.

†Adjusted statistical significance level, $\mathrm{p}<0.01$; statistically significant results are in bold.

$\ddagger$ All comparisons are Bonferroni-adjusted; classification of effect sizes: italics: small if $0.01 \leq$ partial $\eta^{2}<0.06$; underlined: medium if $0.06 \leq$ partial $\eta^{2}<0.14$; bold: large if partial $\eta^{2} \geq 0.14$.

BAI, Beck Anxiety Inventory; BDI, Beck Depression Inventory; HRQoL, health-related quality of life; PHQ-15, Patient Health Questionnaire; SF-12, Short-Form Health Survey; VHQ, Vertigo Handicap Questionnaire; VSS, Vertigo Symptom Scale.

between anxiety and balance disorders. ${ }^{29}$ Patients with phobic/ anxiety and vestibular disorders frequently exhibit avoidance behaviour and report dizziness, spatial disorientation and anxiety in particular environments. ${ }^{30}$ Increased visual dependence (ie, the preferential use of vision for spatial orientation and postural control) and subsequent increased body sway can be observed in patients with primary vestibular disorders and those with phobic/anxiety disorders. ${ }^{31}{ }^{32}$ However, upon a closer look, increased body sway differed in patients with vestibular disorders from those with phobic/anxiety disorders. In patients with vestibular disorders, the body sway increased in more difficult balance tasks, whereas in patients with phobic disorders, it turned out that the more difficult the balance task was, the better the balance performance occurred. ${ }^{33}$ Thus, 'instability' measured in patients with phobia may just be a matter of postural strategy rather than postural capability. Although the term 'phobic postural vertigo' is not a recognised DSM-IV/V or International Classification of Diseases-10 diagnosis, it is a clinically used concept describing a subgroup of patients with certain characteristics, such as dizziness and postural imbalance in particular situations (eg, in shopping centres) and with avoidance behaviour. ${ }^{34}$ More generally, it has been suggested that the association between vertigo/dizziness and anxiety may be explained by neuroanatomical connections between the vestibular system and neuronal pathways that are involved in anxiety/ phobic conditioning and modulated by monoaminergic and noradrenergic influences. ${ }^{29}$

We also found that affective disorders were most frequent in patients with BPPV, central vertigo or multisensory deficits. These vertigo/dizziness disorders are typically observed in older patients and are often associated with feelings of depression. ${ }^{35}$ Furthermore, somatoform disorders were mainly diagnosed in patients with vestibular migraine, which agrees with previous studies. ${ }^{6} 36$

A second major finding in the present study was that nearly half of the patients who were referred to a specialised, interdisciplinary vertigo/dizziness treatment centre had a current psychiatric comorbidity according to the SCID-I, which is the gold standard for diagnosing mental disorders and was conducted blind to patient vestibular diagnosis. ${ }^{37}$ In agreement with previous studies, ${ }^{4} 11$ we found that anxiety and phobic disorders were most frequent, followed by somatoform and affective disorders. The prevalence of psychiatric disorders was significantly higher in the non-organic vertigo/dizziness subgroup than in the organic subgroup. Our results also support our second hypothesis, as patients with psychiatric comorbidity reported more psychosocial impairment, including more vertigo-related symptoms, greater autonomic arousal, more depressive, anxiety and somatisation symptoms, and lower psychological HRQoL, as compared with patients without psychiatric comorbidity. With 
respect to vertigo-related handicaps, the worst combination was having non-organic vertigo/dizziness and psychiatric comorbidity. These differences in self-reported complaints and symptoms underscore the impairment and handicaps experienced by people with vertigo/dizziness and psychiatric comorbidity. ${ }^{4}$ In longitudinal studies, patients with mixed physical and psychological symptoms were found to be at risk of remaining symptomatic and handicapped. ${ }^{38}$ However, we found no difference between subgroups in physical HRQoL as measured by the SF-12. Vertigo/dizziness-related QoL measures, however, may be more sensitive and reliable than general physical HRQoL measures, such as the SF-12, as the former measures reflect the specific impact of vertigo/dizziness and associated symptoms.

Given the associations among vertigo/dizziness, comorbid psychiatric disorders and self-reported impairment that may lead to high costs for the healthcare and social systems, it is important to identify and effectively treat these patients early. ${ }^{8}{ }^{11}$ Our findings illustrate the relevance of an interdisciplinary approach when diagnosing patients with vertigo/dizziness. Psychological counselling and psychotherapy should be included in the treatment plan of these patients to address the predominant psychiatric problem, as there is preliminary evidence that psychotherapy may be effective in patients with medically unexplained vertigo/dizziness. ${ }^{39}$

Our results are limited because the SCID-I does not consider the aetiology of psychiatric disorders (ie, whether they are caused by vertigo/dizziness). Although all interviewers underwent intensive training and regularly attended supervision meetings, and SCID inter-rater reliability is typically high, there may have been systematic overdiagnosis or underdiagnosis as compared with standard norms. Further, the study design was cross-sectional, which does not allow for causal conclusions. The vestibular paroxysmia group may also be slightly contaminated by patients with non-organic dizziness, as this diagnosis was based on patient history, positive MRI for neurovascular cross-compression and response to treatment with carbamazepine (ie, diagnostic criteria as defined by Brandt and Dieterich and Hüfner et al). ${ }^{18} 19$ The MRI criterion (ie, cross-compression) is very sensitive ( $<5 \%$ false negative) but not very specific ( $>25 \%$ false positive) ${ }^{40}$ and in addition, response to carbamazepine could be a placebo response. Thus, there may be in some cases a slight overlap between the 'organic' and 'non-organic' groups. Furthermore, according to the definition of somatoform disorders in the DSM-IV (SCID-I) we used the term 'medically unexplained vertigo/dizziness' though it may be problematic, particularly in regard to the concept of the Somatic Symptom Disorder of the DSM-V. However, as the study began in 2010, we used the SCID-I, which is based on the DSM-IV-the valid diagnostic manual of that time. Our sample was unselected and representative of a specialised tertiary care department, rather than composed of all patients with vertigo/dizziness. As most patients who present in tertiary care were previously treated by neurologists in secondary care settings, and as only patients who do not adequately respond to previous treatment approaches are referred to tertiary care, a selection bias must be assumed (ie, some dizziness syndromes are missing). Nevertheless, recruitment within a specialised tertiary care department allowed us to include and investigate a wide range of organic vertigo/dizziness subgroups, such as vestibular paroxysmia, which had never been investigated until now. Additional strengths of this study are that we examined a large sample of patients and used extremely high international diagnostic standards. Different diagnostic approaches were also used, including clinical-neurological examinations by experts at a tertiary referral centre, structured interviews for assessing psychiatric disorders by trained clinical staff, and patient self-report questionnaires concerning psychosocial variables that were evaluated in light of diagnostic subgroups.

In conclusion, our study supports previous findings that many patients suffering from vertigo/dizziness also exhibit comorbid psychiatric disorders, particularly anxiety and phobic disorders. ${ }^{6}$ In particular, we identified vestibular paroxysmia as an especially vulnerable subgroup for the occurrence of comorbid psychiatric disorders. Patients with vestibular migraine, vestibular paroxysmia or Meniere's disease were frequently affected by anxiety/ phobic disorders, which may be explained by the uncontrollability of vertigo/dizziness attacks in these conditions. ${ }^{28}$ Patients with psychiatric comorbidity reported more psychosocial impairment and greater handicaps as compared with patients without psychiatric comorbidity, indicating the urgent need for the early and effective detection and treatment of these patients. ${ }^{39}{ }^{41}$ Further research should focus on risk factors for the development of psychiatric comorbidity subsequent to organic vertigo/dizziness. Also, considering the high prevalence rate of psychiatric comorbidity and the substantial impairment and handicaps in patients with vertigo/dizziness, an internationally accepted diagnostic classification that describes all aspects of patients with psychiatric comorbidity should be developed and implemented.

Contributors All authors made substantial contributions to this study. CL designed the study and contributed to the final draft of the manuscript. PH and MD designed the study and contributed to the final version of the manuscript. TB provided conceptual advice and contributed to the final version of the manuscript. MS and $\mathrm{KJ}$ recruited patients, performed data assessment and contributed to the final version of the manuscript. AE-H contributed to the final version of the manuscript. RF recruited patients and contributed to the final version of the manuscript. $A D$ contributed to the final draft of the manuscript. GS designed the study, conducted statistical analysis and wrote the manuscript.

Funding Parts of this project were supported by funding from the German Federal Ministry of Education and Research (01 EO 0901). The authors bear full responsibility for the content of this publication.

Competing interests KJ has received speaker's honoraria from Abbott, Alkem, Boehringer Ingelheim and Medtronic and research support from Biogen Idec. MS is Joint Chief Editor of the Journal of Neurology, Editor-in-Chief of Frontiers in Neuro-otology, and Section Editor of F1000. He has received speaker's honoraria from Abbott, UCB, GSK, Teva, Biogen Idec, Pierre-Fabre, Eisai and Hennig Pharma. $\mathrm{CL}$ has received speaker's honoraria from Heel and royalties from Abbott and Heel.

Patient consent Obtained.

Ethics approval This study was approved by the Ethics Committee of the University of Munich (ref. 108-10).

Provenance and peer review Not commissioned; externally peer reviewed.

\section{REFERENCES}

1 Neuhauser HK. Epidemiology of vertigo. Curr Opin Neurol 2007;20:40-6.

2 Tusa RJ. Dizziness. Med Clin North Am 2009;93:263-71, vii.

3 de Waal MW, Arnold IA, Eekhof JA, et al. Somatoform disorders in general practice: prevalence, functional impairment and comorbidity with anxiety and depressive disorders. Br J Psychiatry 2004;184:470-6.

4 Eckhardt-Henn A, Breuer P, Thomalske C, et al. Anxiety disorders and other psychiatric subgroups in patients complaining of dizziness. J Anxiety Disord 2003;17:369-88.

5 Best C, Eckhardt-Henn A, Diener G, et al. Interaction of somatoform and vestibular disorders. J Neurol Neurosurg Psychiatry 2006;77:658-64.

6 Best C, Eckhardt-Henn A, Tschan R, et al. Psychiatric morbidity and comorbidity in different vestibular vertigo syndromes. Results of a prospective longitudinal study over one year. J Neurol 2009;256:58-65.

7 Eckhardt-Henn A, Best C, Bense $\mathrm{S}$, et al. Psychiatric comorbidity in different organic vertigo syndromes. J Neurol 2008;255:420-8.

8 Tschan R, Best C, Beutel ME, et al. Patients' psychological well-being and resilient coping protect from secondary somatoform vertigo and dizziness (SVD) 1 year after vestibular disease. J Neurol 2011;258:104-12.

9 Godemann F, Schabowska A, Naetebusch B, et al. The impact of cognitions on the development of panic and somatoform disorders: a prospective study in patients with vestibular neuritis. Psychol Med 2006;36:99-108. 
10 Yardley L, Beech S, Weinman J. Influence of beliefs about the consequences of dizziness on handicap in people with dizziness, and the effect of therapy on beliefs. J Psychosom Res 2001;50:1-6.

11 Wiltink J, Tschan R, Michal M, et al. Dizziness: anxiety, health care utilization and health behavior-results from a representative German community survey. J Psychosom Res 2009;66:417-24.

12 Wittchen H-U, Wunderlich U, Gruschwitz S, et al. SCID-I: structured clinical interview for DSM-IV. Axis I: mental disorders. Interview book. Göttingen: Hogrefe, 1997.

13 Lahmann C, Henningsen P, Dieterich M, et al. The Munich Diagnostic and Predictor Study of Dizziness: objectives, design, and methods. J Neurol 2012;259:702-11.

14 Brandt T, Dieterich M, Strupp M. Vertigo and dizziness: common complaints. 2nd edn. London: Springer, 2013

15 Radtke A, Neuhauser $H$, von Brevern $M$, et al. Vestibular migraine-validity of clinical diagnostic criteria. Cephalalgia 2011;31:906-13.

16 Neuhauser $\mathrm{H}$, Leopold $\mathrm{M}$, von Brevern $\mathrm{M}$, et al. The interrelations of migraine, vertigo, and migrainous vertigo. Neurology 2001;56:436-41.

17 American Academy of Otolaryngology Head and Neck Surgery. Committee on Hearing and Equilibrium guidelines for the diagnosis and evaluation of therapy in Meniere's disease. American Academy of Otolaryngology-Head and Neck Foundation, Inc. Otolaryngol Head Neck Surg 1995;113:181-5.

18 Brandt T, Dieterich M. Vestibular paroxysmia: vascular compression of the eighth nerve? Lancet 1994;343:798-9.

19 Hüfner K, Barresi D, Glaser M, et al. Vestibular paroxysmia: diagnostic features and medical treatment. Neurology 2008;71:1006-14.

20 Tschan R, Wiltink J, Best C, et al. Validation of the German version of the Vertigo Handicap Questionnaire (VHQ) in patients with vestibular vertigo syndromes or somatoform vertigo and dizziness. Psychother Psychosom Med Psychol 2009;59:1-12.

21 Tschan R, Wiltink J, Best C, et al. Validation of the German version of the Vertigo Symptom Scale (VSS) in patients with organic or somatoform dizziness and healthy controls. J Neurol 2008;255:1168-75.

22 Kuhner C, Burger C, Keller F, et al. Reliability and validity of the Revised Beck Depression Inventory (BDI-II). Results from German samples. Nervenarzt 2007;78:651-6.

23 Margraf J, Ehlers A. Beck anxiety inventory, original version by A.T. Beck and R.A. Stern. Pearson Assessment, 2007

24 Gräfe K, Zipfel S, Herzog W, et al. Screening for psychiatric disorders with the Patient Health Questionnaire (PHQ). Results from the German validation study. Diagnostica 2004;50:171-81.
25 Bullinger M, Morfeld M, Kohlmann T, et al. SF-36 health survey in rehabilitation research. Findings from the North German Network for Rehabilitation Research, NVRF, within the rehabilitation research funding program. Rehabilitation (Stuttg) 2003:42:218-25

26 Cohen J. Statistical power analysis for the behavioural sciences. Hillsdale: Lawrence Erlbaum Associates, 1988.

27 Tabachnick BG, Fidell LS. Using multivariate statistics. Boston: Pearson Education, 2007.

28 Kirby SE, Yardley L. Physical and psychological triggers for attacks in Meniere's disease: the patient perspective. Psychother Psychosom 2012;81:396-8.

29 Furman JM, Balaban CD, Jacob RG, et al. Migraine-anxiety related dizziness (MARD): a new disorder? J Neurol Neurosurg Psychiatry 2005;76:1-8.

30 Beidel DC, Horak FB. Behavior therapy for vestibular rehabilitation. J Anxiety Disord 2001;15:121-30.

31 Jacob RG, Redfern MS, Furman JM. Space and motion discomfort and abnorma balance control in patients with anxiety disorders. J Neurol Neurosurg Psychiatry 2009;80:74-8.

32 Bronstein AM. Under-rated neuro-otological symptoms: Hoffman and Brookler 1978 revisited. Br Med Bull 2002;63:213-21.

33 Querner V, Krafczyk S, Dieterich M, et al. Patients with somatoform phobic postural vertigo: the more difficult the balance task, the better the balance performance. Neurosci Lett 2000;285:21-4.

34 Brandt T. Phobic postural vertigo. Neurology 1996;46:1515-9.

35 Wetmore SJ, Eibling DE, Goebel JA, et al. Challenges and opportunities in managing the dizzy older adult. Otolaryngol Head Neck Surg 2011;144:651-6.

36 Jahn K, Dieterich M. Recent advances in the diagnosis and treatment of balance disorders. J Neurol 2011;258:2305-8.

37 Ventura J, Liberman RP, Green MF, et al. Training and quality assurance with the Structured Clinical Interview for DSM-IV (SCID-I/P). Psychiatry Res 1998;79:163-73.

38 Yardley L. Overview of psychologic effects of chronic dizziness and balance disorders. Otolaryngol Clin North Am 2000;33:603-16.

39 Schmid G, Henningsen P, Dieterich M, et al. Psychotherapy in dizziness: a systematic review. J Neurol Neurosurg Psychiatry 2011;82:601-6.

40 Best C, Gawehn J, Krämer HH, et al. MRI and neurophysiology in vestibular paroxysmia: contradiction and correlation. J Neurol Neurosurg Psychiatry 2013:84:1349-56.

41 Sattel H, Lahmann C, Gundel H, et al. Brief psychodynamic interpersonal psychotherapy for patients with multisomatoform disorder: randomised controlled trial. Br J Psychiatry 2012;200:60-7. 

impairment among patients with vertigo and dizziness

Claas Lahmann, Peter Henningsen, Thomas Brandt, Michael Strupp, Klaus Jahn, Marianne Dieterich, Annegret Eckhardt-Henn, Regina Feuerecker, Andreas Dinkel and Gabriele Schmid

J Neurol Neurosurg Psychiatry 2015 86: 302-308 originally published online June 24, 2014

doi: 10.1136/jnnp-2014-307601

Updated information and services can be found at:

http://jnnp.bmj.com/content/86/3/302

\section{These include:}

Supplementary Material

References

Email alerting service
Supplementary material can be found at:

http://jnnp.bmj.com/content/suppl/2014/06/24/jnnp-2014-307601.DC1

This article cites 36 articles, 8 of which you can access for free at: http://jnnp.bmj.com/content/86/3/302\#BIBL

Receive free email alerts when new articles cite this article. Sign up in the box at the top right corner of the online article.

Topic
Collections

Articles on similar topics can be found in the following collections

Drugs: CNS (not psychiatric) (1945)

Ear, nose and throat/otolaryngology (208)

Stroke (1449)

Headache (including migraine) (459)

Mood disorders (including depression) (221)

\section{Notes}

To request permissions go to:

http://group.bmj.com/group/rights-licensing/permissions

To order reprints go to:

http://journals.bmj.com/cgi/reprintform

To subscribe to BMJ go to:

http://group.bmj.com/subscribe/ 